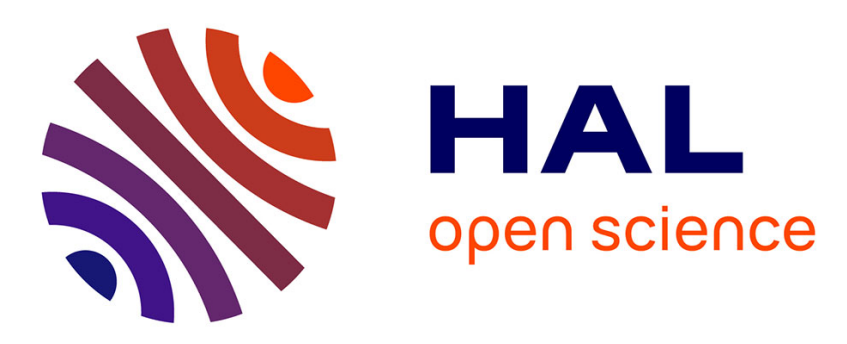

\title{
Sub-Poissonian atom-number fluctuations using light-assisted collisions
}

Yvan R. P. Sortais, A. Fuhrmanek, R. Bourgain, Antoine Browaeys

\section{To cite this version:}

Yvan R. P. Sortais, A. Fuhrmanek, R. Bourgain, Antoine Browaeys. Sub-Poissonian atom-number fluctuations using light-assisted collisions. Physical Review Online Archive (PROLA), 2012, 85, pp.035406. 10.1103/PhysRevA.85.035403 . hal-00685861

\section{HAL Id: hal-00685861 https://hal.science/hal-00685861}

Submitted on 12 Jan 2015

HAL is a multi-disciplinary open access archive for the deposit and dissemination of scientific research documents, whether they are published or not. The documents may come from teaching and research institutions in France or abroad, or from public or private research centers.
L'archive ouverte pluridisciplinaire HAL, est destinée au dépôt et à la diffusion de documents scientifiques de niveau recherche, publiés ou non, émanant des établissements d'enseignement et de recherche français ou étrangers, des laboratoires publics ou privés. 


\title{
Sub-Poissonian atom-number fluctuations using light-assisted collisions
}

\author{
Y. R. P. Sortais, A. Fuhrmanek, R. Bourgain, and A. Browaeys \\ Laboratoire Charles Fabry, Institut d'Optique, CNRS, Univ Paris-Sud, 2 Avenue Augustin Fresnel, 91127 Palaiseau Cedex, France
}

(Received 22 November 2011; published 13 March 2012)

\begin{abstract}
We investigate experimentally the number statistics of a mesoscopic ensemble of cold atoms in a microscopic dipole trap loaded from a magneto-optical trap and find that the atom-number fluctuations are reduced with respect to a Poisson distribution due to light-assisted two-body collisions. For numbers of atoms $N \gtrsim 2$, we measure a reduction factor (Fano factor) of $0.72 \pm 0.07$, which differs from 1 by more than four standard deviations. We analyze this fact by a general stochastic model describing the competition between the loading of the trap from a reservoir of cold atoms and multiatom losses, leading to a master equation. Applied to our experimental regime, this model indicates an asymptotic value of 3/4 for the Fano factor at large $N$ and in the steady state. We thus show that we have reached the ultimate level of reduction in number fluctuations in our system.
\end{abstract}

DOI: 10.1103/PhysRevA.85.035403

PACS number(s): 34.50.Rk, 03.65.Ta, 05.40.-a, 42.50.Lc

There is a growing interest in the study of mesoscopic systems containing between 10-100 particles. For example, mesoscopic ensembles of ultracold atoms could be a useful tool for quantum-information processing (e.g., Refs. [1,2]) and for precision measurements beyond the standard quantum limit $[3,4]$. They are also a test bed for the investigation of many-body correlated quantum systems [5] and for the study of collective effects such as super-radiance (e.g., Ref. [6]). All these applications require precise knowledge of the distribution of the number of atoms as the properties of these finite-size samples are governed by their statistical nature. In particular, the knowledge of the variance of the number distribution is important. In this paper we show experimentally and theoretically that one of the conceptually simplest mesoscopic systems, namely, a few cold atoms in a tight dipole trap being loaded from a cold atomic cloud [7-9], already exhibits nontrivial sub-Poissonian statistics.

The preparation of an atomic sample with a non-Poissonian atom-number distribution requires a nonlinear mechanism usually provided by interactions between ultracold atoms. For example, the dispersive $s$-wave interaction was used to reduce the relative atom-number fluctuations between the sites of a two-well potential $[10,11]$ and of an optical lattice [12]. This led in particular to the study of the Mott transition [13,14]. This interaction was also used to demonstrate reduced atom-number fluctuations in a single tight dipole trap [15]. Recently, the production of a sample with definite atom numbers was demonstrated using the Pauli blockade [16].

Inelastic collisions between ultracold atoms can also provide the nonlinearity required to modify the atom-number statistics as shown recently $[17,18]$. In those experiments, three-body inelastic collisions induce losses in an initially trapped sample of 50-300 atoms at or close to quantum degeneracy, and the ever decreasing fraction of remaining atoms exhibits reduced number fluctuations with respect to a Poisson distribution. Here, we consider theoretically a different and yet more general regime in which the trap continuously experiences the interplay between a loading process from a reservoir of laser-cooled atoms and strong inelastic $\rho$-body losses $(\rho \geqslant 1)$, and we investigate experimentally the case $\rho=2$ in which the losses are due to light-assisted collisions. This situation is used elsewhere to, e.g., produce a single-atom source $[19,20]$. There, one operates in the "collisional-blockade" regime where the loading rate is such that the microscopic trap contains one or zero atoms with equal probabilities $(\langle N\rangle=0.5)$ and the atom-number distribution is maximally sub-Poissonian with variance $\Delta N^{2}=0.5\langle N\rangle[21]$.

Here, we explore the regime where $\langle N\rangle$ goes beyond 0.5 in the steady state as we increase the loading rate. In practice, we prepare a thermal ensemble of up to 10 cold atoms at a temperature of $\sim 100 \mu \mathrm{K}$ in a microscopic dipole trap. We observe that the atom-number distribution remains sub-Poissonian and that the reduction in number fluctuations with respect to the Poisson distribution $\Delta N^{2} /\langle N\rangle$ is locked to a constant value of 0.75 for $\langle N\rangle \gtrsim 2$, a fact that has been overlooked so far. To explain this fact, we use a microscopic approach that takes into account the stochastic nature of the competing loading and loss processes, and we calculate the atom-number distribution at any time of the system evolution. We do so by solving a master equation both numerically and analytically and find good agreement with the average result of a Monte Carlo approach with which we study the individual behavior of atoms. Using this general theoretical approach, we analyze our data and find that we have reached experimentally the ultimate level of reduction in atom-number fluctuations that one can expect in a dipole trap operating in our regime $(\rho=2)$. The formalism presented in this paper is applicable to any system in which a random-loading process competes with a $\rho$-body-loss process, whatever its nature and whatever the number of atoms.

To study the number statistics of a few atoms in the presence of competing random processes, we implemented the following experiment (details can be found in Ref. [9]). First, we produced a microscopic optical-dipole trap at $850 \mathrm{~nm}$ by sharply focusing a laser beam [22]. We then loaded this trap from a magneto-optical trap (MOT) of ${ }^{87} \mathrm{Rb}$ atoms surrounding the region of the dipole trap. Atoms enter the dipole trap randomly, are trapped thanks to the cooling effect of the MOT beams, and are expelled from the trap due mainly to inelastic two-body collisions assisted by the near-resonant light of the cooling beams and, to a minor extent, to collisions with the residual background gas in the chamber (one-body losses). We measured elsewhere the two-bodyand one-body-loss constants to be $\beta^{\prime} \sim 500$ (at.s) ${ }^{-1}$ and 


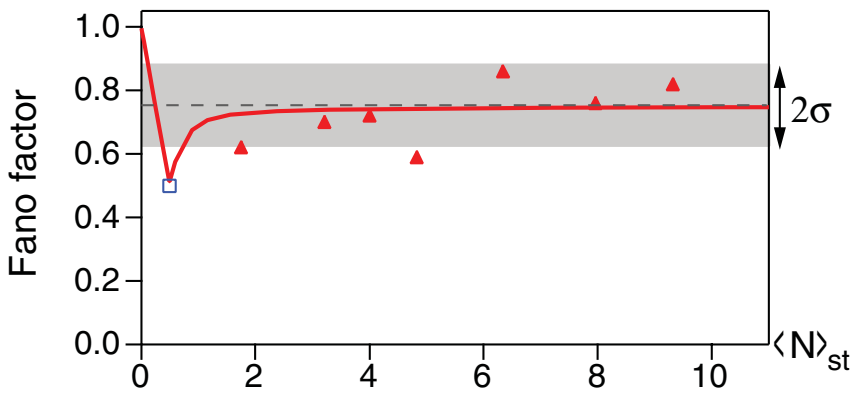

FIG. 1. (Color online) Fano factor $F$ versus the average number of atoms $\langle N\rangle_{\mathrm{st}}$ in the microscopic dipole trap in the steady state. The square and triangles are the experimental data collected in the collisional-blockade regime $\left(\langle N\rangle_{\mathrm{st}}=0.5\right)$ and beyond $\left(\langle N\rangle_{\mathrm{st}}>0.5\right)$, respectively. The solid line is the model based on a stochastic process (see text) with $\beta^{\prime}=500$ (at.s) ${ }^{-1}$ and $\gamma=0.2 \mathrm{~s}^{-1}$. The dashed line is the theoretical limit $F=3 / 4$ for $\langle N\rangle_{\mathrm{st}} \gg 1 . \sigma$ is the rms dispersion of the data collected beyond the collisional-blockade regime.

$\gamma \sim 0.2 \mathrm{~s}^{-1}$, respectively [23]. The actual value of the loading rate $R$ is proportional to the MOT local density in the vicinity of the microscopic trap, which is the parameter that we vary. For values of $R \gg \beta^{\prime}$, the mean number of trapped atoms in the steady state exceeds unity and is $\langle N\rangle_{\mathrm{st}}=\sqrt{R / \beta^{\prime}}$ while for $R \ll \gamma$ it goes to zero as $\langle N\rangle_{\text {st }}=R / \gamma$. The intermediate regime corresponds to the collisional-blockade regime where $\langle N\rangle_{\mathrm{st}}=0.5$. Experimentally, we operate at $\langle N\rangle_{\mathrm{st}} \gtrsim 2$ in the following.

To get information on the number distribution of atoms in the dipole trap in the steady state, we release the atoms from the trap and probe them with a pulse of resonant light. Using an intensifier to amplify single-photon events above the noise of our CCD camera, we count the detected fluorescence photons individually [9]. This number is proportional, on average, to the number of atoms $N$ in the trap before release. We build up the number distribution of counted photons by repeating this loading and probing experiment about 100 to 1000 times. Knowing the response of our imaging system to one atom exactly, we extract from the photon distribution the mean $\langle N\rangle_{\mathrm{st}}$ and the variance $\Delta N^{2}$ of the atom-number distribution in the steady state and calculate the corresponding Fano factor $F=$ $\Delta N^{2} /\langle N\rangle_{\mathrm{st}}$. The data shown in Fig. 1 indicate a clear reduction of the atom-number fluctuations with respect to a Poisson distribution for $\langle N\rangle_{\text {st }} \gtrsim 2$ with a mean $F=0.72$ and a total uncertainty (one standard deviation) of 0.07 . This uncertainty is obtained by adding quadratically the statistical (type A) uncertainty of 0.05 (deduced from the rms dispersion of the data) and the systematic (type B) uncertainty of 0.04 , which we estimated in a previous work [9].

Qualitatively, this reduction can be understood as follows. If the losses were governed by random one-body events, e.g., background gas collisions, the trade-off between the random loading of the trap and the losses would result in a Poisson distribution with a mean atom number $\langle N\rangle_{\text {st }}$ in the steady state. If the losses now involve higher-body processes $(\rho \geqslant$ 2 ), the loss rate varies as the number of $\rho$ uplets in the $N$ atom ensemble, i.e., increases nonlinearly with $N$. For a given mean atom number, this leads to the number distribution being narrower than a Poisson distribution as the losses are more efficient on the high- $N$ side of the distribution.

To explain quantitatively the sub-Poissonian behavior of the atom-number distribution, we use the following stochastic model that takes into account the three random processes involved, i.e., the loading, the two-body losses, and the one-body losses. We consider the evolution in time of the probability $p_{N}(t)$ to have $N$ atoms in the dipole trap. To calculate the probability $p_{N}(t+d t)$, we sum the contributions of all channels associated with the random processes mentioned above that lead to having $N$ atoms in the trap at $t+d t$, given that the trap could possibly be filled with either $N-1, N$, $N+1$, or $N+2$ atoms at time $t$. The probability that a loading event occurs in the time interval $d t$ when there are already $N$ atoms in the trap is $R d t p_{N}(t)$. Similarly, the probability that a loss event occurs during $d t$ is $\gamma N d t p_{N}(t)$ for one-body events and $\beta^{\prime} \frac{N(N-1)}{2} d t p_{N}(t)$ for two-body events. We obtain eventually the following equation:

$$
\begin{aligned}
p_{N}(t+d t)= & p_{N}(t)\left\{1-\left[R+\gamma N+\beta^{\prime} \frac{N(N-1)}{2}\right] d t\right\} \\
& +p_{N-1}(t) R d t+p_{N+1}(t) \gamma(N+1) d t \\
& +p_{N+2}(t) \beta^{\prime} \frac{(N+2)(N+1)}{2} d t
\end{aligned}
$$

Taking the limit $d t \rightarrow 0$, Eq. (1) yields the following master equation that rules the evolution of $p_{N}(t)$ in time:

$$
\begin{aligned}
\frac{d p_{N}}{d t}= & R\left(\mathbb{E}^{-1}-\mathbb{1}\right)\left[p_{N}\right]+\gamma(\mathbb{E}-\mathbb{1})\left[N p_{N}\right] \\
& +\beta^{\prime}\left(\mathbb{E}^{2}-\mathbb{1}\right)\left[\frac{N(N-1)}{2} p_{N}\right],
\end{aligned}
$$

where $\mathbb{E}$ is the "step operator" defined by its effect on an arbitrary function $f(N)$

$$
\mathbb{E}[f(N)]=f(N+1), \quad \mathbb{E}^{-1}[f(N)]=f(N-1),
$$

and $\mathbb{1}$ is the identity operator. Using Eq. (2), we obtain the equation of evolution of the mean number of atoms $\langle N\rangle=$ $\sum_{N=0}^{\infty} N p_{N}$ :

$$
\frac{d\langle N\rangle}{d t}=R-\gamma\langle N\rangle-\beta^{\prime}\langle N\rangle(\langle N\rangle-1)-\beta^{\prime} \Delta N^{2} .
$$

When $\Delta N^{2}=0$, we recover the phenomenological equation sometimes used to describe the loading of a trap containing a small number of atoms [21,24], i.e., $d N / d t=R-\gamma N-$ $\beta^{\prime} N(N-1)$. When $\Delta N^{2}=\langle N\rangle$ (i.e., assuming a Poisson distribution), Eq. (4) also yields the widely used equation $d\langle N\rangle / d t=R-\gamma\langle N\rangle-\beta^{\prime}\langle N\rangle^{2}$. However, without any $a$ priori relation between $\Delta N^{2}$ and $\langle N\rangle$, Eq. (4) cannot be solved analytically.

To calculate the first moments of the number distribution, we used three different approaches. First, we solved numerically Eq. (2) using the boundary conditions $p_{N}(0)=$ $\delta_{N, 0}$ and, for $N \gg\langle N\rangle_{\mathrm{st}}, p_{N}(t)=0$. As an example, Fig. 2 illustrates the time evolution of the probabilities $p_{N}(t)$ for parameters leading to $\langle N\rangle_{\mathrm{st}}=3.6$. The number distribution is found to be sub-Poissonian with $F=0.74$. By varying the loading rate, the same approach yields the distribution for any value of $\langle N\rangle_{\mathrm{st}}$. We analyze the case where $\gamma \ll \beta^{\prime}$ in the following. When $\langle N\rangle_{\text {st }} \ll 0.5$, we find, as expected, that 


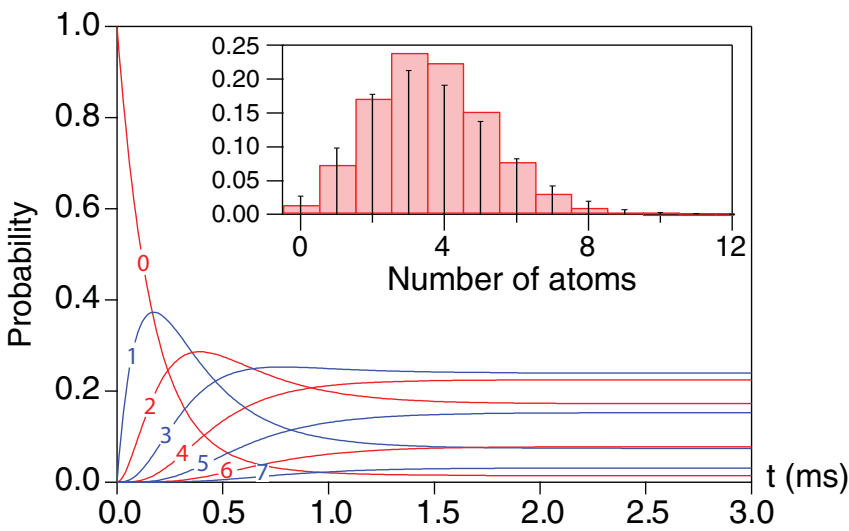

FIG. 2. (Color online) Set of numerical solutions $\left\{p_{N}(t)\right\}$ of Eq. (2), labeled by $N$. The parameters $R=6000 \mathrm{~s}^{-1}, \gamma=0.2 \mathrm{~s}^{-1}$, and $\beta^{\prime}=500$ (at.s) $)^{-1}$ lead to $\langle N\rangle_{\mathrm{st}}=3.6 \sim \sqrt{R / \beta^{\prime}}$. Inset: The set of solutions in the steady state $\left\{p_{N}(t=3 \mathrm{~ms})\right\}$ (filled bars) is clearly sub-Poisson, and the sticks indicate the Poisson distribution with the same mean value.

the distribution is close to a Poisson law as one-body losses then dominate two-body losses [see Fig. 3(a)]. ${ }^{1}$ The presence of two-body-processes-induced losses of atom pairs leads to a sub-Poissonian behavior that is maximal for $\langle N\rangle_{\mathrm{st}}=0.5$, corresponding to $p_{0}=p_{1}=0.5$. While this regime has been described before (see Ref. [21]), the numerical approach predicts that atom-number fluctuations do not become Poissonian for larger numbers of atoms. In fact, the Fano factor reaches an asymptotic value of 0.75 as soon as $\langle N\rangle_{\text {st }} \gtrsim 2$, corresponding to a reduction of $-1.25 \mathrm{~dB}$ with respect to the Poisson case. The numerical prediction reproduces well our data as shown in Fig. 1.

The second approach to solve Eq. (2) is analytical. It is valid for $\langle N\rangle_{\mathrm{st}} \gg 1$ only and follows closely the approach of Ref. [25]. We first rewrite the master equation into a dimensionless rate equation

$$
\begin{aligned}
\frac{d p_{N}}{d \tau}= & \langle N\rangle_{\mathrm{st}}\left(\mathbb{E}^{-1}-\mathbb{1}\right)\left[p_{N}\right] \\
& +\frac{1}{\langle N\rangle_{\mathrm{st}}}\left(\mathbb{E}^{2}-\mathbb{1}\right)\left[\frac{N(N-1)}{2} p_{N}\right],
\end{aligned}
$$

where the one-body-loss term of Eq. (2) has been neglected (following $R \gg \beta^{\prime} \gg \gamma$ ) and $\tau=t \sqrt{R \beta^{\prime}}$ is a dimensionless time variable. Since the number distribution in the steady state is expected to peak around $\langle N\rangle_{\mathrm{st}}$ with a width on the order of $\sqrt{\langle N\rangle_{\mathrm{st}}}$, we consider the number of trapped atoms at time $\tau$ as a stochastic quantity of the form

$$
N(\tau)=\langle N\rangle_{\mathrm{st}} \phi(\tau)+\sqrt{\langle N\rangle_{\mathrm{st}}} \xi(\tau),
$$

where $\xi(\tau)$ is a stochastic variable with mean $\langle\xi\rangle(\tau)=0$ and an amplitude of $\sim 1$ and $\phi(\tau)$, also on the order of 1 , is a deterministic and slowly varying function of time $\left[\phi(\tau)=\langle N\rangle(\tau) /\langle N\rangle_{\mathrm{st}}\right]$. We then consider the probability

\footnotetext{
${ }^{1}$ The distribution would remain Poissonian for any values of $\langle N\rangle_{\mathrm{st}}$ in the absence of two-body losses $\left(\beta^{\prime}=0\right)$ as can be derived analytically from Eq. (2).
}
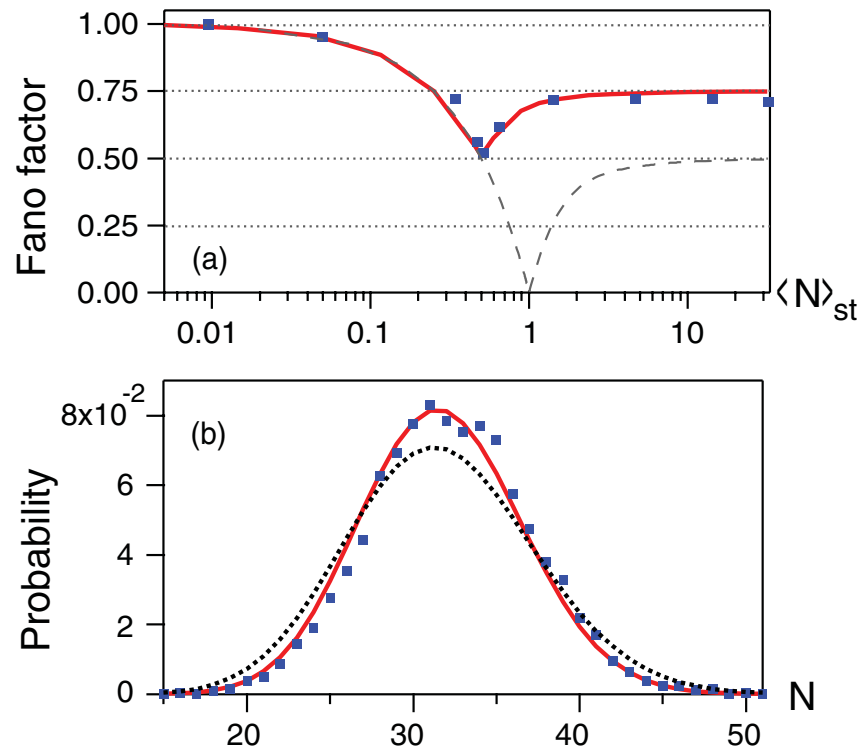

FIG. 3. (Color online) Theoretical results obtained by different approaches. The solid line is the numerical solution of Eq. (2) for

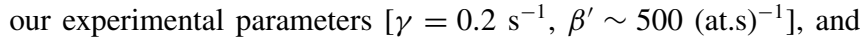
the squares are the Monte Carlo simulation. (a) Dependence of the Fano factor on $\langle N\rangle_{\mathrm{st}}$. (b) Example of the calculated atom-number distribution in the steady state with $R=5 \times 10^{5} \mathrm{~s}^{-1}$, yielding $\langle N\rangle_{\mathrm{st}}=$ 32. The numerical solution is indistinguishable from the Gaussian solution of Eq. (8). We find a Fano factor $F=3 / 4$. The dotted line is the Poisson distribution with the same mean value. The dashed line in (a) is the numerical solution of Eq. (2) when two-body processes induce the loss of one atom only from the trap [the parameters are $\gamma=5 \times 10^{-3} \mathrm{~s}^{-1}$ and $\beta^{\prime} \sim 500$ (at.s) ${ }^{-1}$ ]. In the limit $\gamma / \beta^{\prime} \rightarrow 0$, $\langle N\rangle_{\mathrm{st}}$ can be locked to 1 in a fully deterministic way $\left(\Delta N^{2}=0\right)$.

$P(\xi, \tau)=p_{N}(\tau)$ that $N$ atoms are in the trap at time $\tau$. Since $p_{N+k}(\tau)=P\left(\xi+\frac{k}{\sqrt{\langle N\rangle_{\mathrm{st}}}}, \tau\right)$ and $\langle N\rangle_{\mathrm{st}} \gg 1$, we replace $p_{N+k}(\tau)$ in Eq. (5) with a Taylor expansion of $P(\xi, \tau)$ in powers of $1 / \sqrt{\langle N\rangle_{\mathrm{st}}}$. Replacing $p_{N}(\tau)$ with $P(\xi, \tau)$, the time derivative $d p_{N} / d \tau$ becomes $\partial_{\tau} P-\sqrt{N_{\mathrm{st}}} \dot{\phi} \partial_{\xi} P{ }^{2}$ Identification of the power terms in the expanded master equation then yields the following equations that rule the evolution of $\phi$ and $P$ in time:

$$
\begin{gathered}
\dot{\phi}=1-\phi^{2}, \\
\partial_{\tau} P=2 \phi \partial_{\xi}(\xi P)+\frac{1}{2}\left(2 \phi^{2}+1\right) \partial_{\xi}^{2} P .
\end{gathered}
$$

Equation (8) is a linear Fokker-Planck equation with timedependent coefficients, the steady-state solution of which is Gaussian [25]. Finally, using Eq. (8), we find that $\left\langle\xi^{2}\right\rangle$ evolves in time according to

$$
\frac{d\left\langle\xi^{2}\right\rangle}{d \tau}=-4 \phi\left\langle\xi^{2}\right\rangle+\left(1+2 \phi^{2}\right)
$$

\footnotetext{
${ }^{2}$ Care must be taken when calculating the total derivative of $P(\xi, \tau)$ with respect to time as Eq. (5) was established with $N$ being held constant during an infinitesimal time interval $d \tau$ so that $\dot{\xi}(\tau)=-\sqrt{\langle N\rangle_{\mathrm{st}}} \dot{\phi}(\tau)$.
} 
Equations (7) and (9) allow us to calculate the evolution of the Fano factor in time, $F(\tau)=\left\langle\xi^{2}\right\rangle(\tau) / \phi(\tau)$. In particular, in the steady state, $\phi=1$, and $F=3 / 4$. This analytical finding is in excellent agreement with our numerical solution (see Fig. 3). Besides, we find that the analytical result is valid for atom numbers as small as $\sim 2$.

Finally, we cross-checked our theoretical results with a Monte Carlo simulation in which we calculated at each time increment the survival probabilities of individual atoms to the various random events involved in the problem [23]. By averaging over many atomic histories, we reconstructed atom-number distributions and found Fano factors in very good agreement with those presented above [see Fig. (3)], validating the master-equation approach.

In conclusion, we discuss our experimental findings and theoretical approaches from a more general perspective. First, the observed reduction in number fluctuations is due to loss terms that vary nonlinearly as $N^{\rho}$ and is thus intrinsically robust to losses (provided $\rho$ and the loading rate remain constant). In our case, we reached experimentally the ultimate level of reduction $(-1.25 \mathrm{~dB})$ predicted by theory when a loading mechanism competes with a two-body nonlinearity, leading to the loss of atom pairs no matter the underlying mechanism (light-assisted collisions, hyperfine-changing collisions, etc.). More generally, the exact level of reduction achievable depends on $\rho$, on the number of atoms being lost after a $\rho$-body process, and on the presence (or the absence) of a loading mechanism. When $R \neq 0$, the analytical approach explained above can be generalized and yields a Gaussian atom-number distribution in the steady state $[P(\xi, \tau)$ evolves according to a Fokker-Planck equation similar to Eq. (8)]. For $\rho$-body processes leading to losses of $\rho$ uplets, one finds an equation similar to Eq. (9) and $F=\frac{1}{2}\left(1+\frac{1}{\rho}\right)$ in the steady state. When $R=0$, slightly better levels of reduction can be achieved as $F=\rho /(2 \rho-1)$. This was recently demonstrated in the case of three-body losses [18]. Finally, we extended our approach to the case in which two-body collisions lead to the loss of one atom only from the trap ${ }^{3}$ as is the case for elastic-collisioninduced evaporative losses and for some light-assisted-loss mechanisms. Such mechanisms have been used recently to produce near-deterministically a single-atom source for quantuminformation processing [26]. Taking these mechanisms into account, our theoretical approach predicts that fluctuations fully vanish when only one atom is left in the trap in the absence of one-body decay [see Fig. 3(a)], i.e., that a robust and fully deterministic preparation of single trapped atoms is in principle possible.

We acknowledge support from the E.U. through the ERC Starting Grant ARENA and from IFRAF and Triangle de la Physique. A.F. acknowledges partial support from the DAAD Doktorandenstipendium. We thank G. Messin and M. P. A. Jones for fruitful discussions.

\footnotetext{
${ }^{3}$ We replaced $\left(\mathbb{E}^{2}-\mathbb{1}\right)$ with $(\mathbb{E}-\mathbb{1})$ in Eq. (2).
}

[1] M. D. Lukin, M. Fleischhauer, R. Cote, L. M. Duan, D. Jaksch, J. I. Cirac, and P. Zoller, Phys. Rev. Lett. 87, 037901 (2001).

[2] M. Saffman, T. G. Walker, and K. Moelmer, Rev. Mod. Phys. 82, 2313 (2010).

[3] D. J. Wineland, J. J. Bollinger, W. M. Itano, F. L. Moore, and D. J. Heinzen, Phys. Rev. A 46, 6797(R) (1992).

[4] V. Giovanetti, S. Lloyd, and L. Maccone, Science 306, 1330 (2004).

[5] I. Bloch, J. Dalibard, and W. Zwerger, Rev. Mod. Phys. 80, 885 (2008).

[6] E. Akkermans, A. Gero, and R. Kaiser, Phys. Rev. Lett. 101, 103602 (2008).

[7] S. Whitlock, R. Gerritsma, T. Fernholz, and R. J. C. Spreeuw, New J. Phys. 11, 023021 (2009).

[8] J. Kruse, C. Gierl, M. Schlosser, and G. Birkl, Phys. Rev. A 81, 060308(R) (2010).

[9] A. Fuhrmanek, Y. R. P. Sortais, P. Grangier, and A. Browaeys, Phys. Rev. A 82, 023623 (2010).

[10] G.-B. Jo, Y. Shin, S. Will, T. A. Pasquini, M. Saba, W. Ketterle, D. E. Pritchard, M. Vengalattore, and M. Prentiss, Phys. Rev. Lett. 98, 030407 (2007).

[11] C. Gross, T. Zibold, E. Nicklas, J. Estève, and M. K. Oberthaler, Nature (London) 464, 1165 (2010).

[12] C. Orzel, A. K. Tuchman, M. L. Fenselau, M. Yasuda, and M. A. Kasevich, Science 291, 2386 (2001).

[13] M. Greiner, O. Mandel, T. Esslinger, T. W. Haensch, and I. Bloch, Nature (London) 415, 39 (2002).
[14] F. Gerbier, S. Fölling, A. Widera, O. Mandel, and I. Bloch, Phys. Rev. Lett. 96, 090401 (2006).

[15] C.-S. Chuu, F. Schreck, T. P. Meyrath, J. L. Hanssen, G. N. Price, and M. G. Raizen, Phys. Rev. Lett. 95, 260403 (2005).

[16] F. Serwane, G. Zürn, T. Lompe, T. B. Ottenstein, A. N. Wenz, and S. Jochim, Science 332, 336 (2011).

[17] A. Itah, H. Veksler, O. Lahav, A. Blumkin, C. Moreno, C. Gordon, and J. Steinhauer, Phys. Rev. Lett. 104, 113001 (2010).

[18] S. Whitlock, C. F. Ockeloen, and R. J. C. Spreeuw, Phys. Rev. Lett. 104, 120402 (2010).

[19] N. Schlosser, G. Reymond, I. Protsenko, and P. Grangier, Nature (London) 411, 1024 (2001).

[20] M. Weber, J. Volz, K. Saucke, C. Kurtsiefer, and H. Weinfürter, Phys. Rev. A 73, 043406 (2006).

[21] N. Schlosser, G. Reymond, and P. Grangier, Phys. Rev. Lett. 89, 023005 (2002).

[22] Y. R. P. Sortais, H. Marion, C. Tuchendler, A. M. Lance, M. Lamare, P. Fournet, C. Armellin, R. Mercier, G. Messin, A. Browaeys, and P. Grangier, Phys. Rev. A 75, 013406 (2007).

[23] A. Fuhrmanek, R. Bourgain, Y. R. P. Sortais, and A. Browaeys, e-print arXiv:1107.5781v2 (submitted to Phys. Rev. Lett.).

[24] B. Ueberholz, S. Kuhr, D. Frese, D. Meschede, and V. Gomer, J. Phys. B 33, L135 (2000).

[25] N. G. van Kampen, Stochastic Processes in Physics and Chemistry, 3rd ed. (Elsevier, Amsterdam, 2007).

[26] T. Grünzweig, A. Hilliard, M. McGovern, and M. F. Andersen, Nat. Phys. 6, 951 (2010). 